\title{
THE 1/3rd SUBHARMONIC AND 3rd SUPERHARMONIC RESONANCE OF A SHAPE MEMORY ALLOY (SMA) LAMINATED BEAM
}

\author{
Xia Hui Zhang ${ }^{1}$ \\ Department of Civil Engineering, Hebei Jiaotong Vocational and Technical College, Shijiazhuang, China \\ Ming $\mathrm{GAO}^{1}$ \\ College of Mechanical and Electronical Engineering, Shandong Agriculture University, Taian, China; \\ Tianjin Key Laboratory of Microgravity and Hypogravity Environment Simulation Technology, Tianjin, China \\ e-mail: gaom@sdau.edu.cn \\ YING $\mathrm{HAO}^{1}$ \\ College of Civil Engineering and Mechanics, Yanshan University, Qinhuangdao, China
}

\begin{abstract}
This paper examines the 1/3rd subharmonic resonance and the 3rd superharmonic resonance of simply-supported shape memory alloy (SMA) laminated beams. First, the dynamic equation for SMA laminated beams under transverse load is established using physical equations, force equilibrium conditions, the compatibility equation of deformation, and a constitutive model of SMA polynomial functions. Then, a differential equation for transverse vibration of the SMA laminated beams is derived by the Galerkin process assuming the boundary conditions for simply-supported beams. Next, the amplitude-frequency response equations for the $1 / 3$ rd subharmonic resonance and the 3rd superharmonic resonance of these beams are derived by an averaging method before their respective transition sets are calculated, and their amplitude-frequency response diagrams were plotted using singularity theory. The results show two different types of amplitude-frequency responses to nonlinear vibration under the 1/3rd subharmonic resonance and the 3rd superharmonic resonance: quasi-linear and hard characteristic. In the quasi-linear area, SMA thickness $A$ does not make much difference to the response of the system, whereas in the hard-characteristics area, under the same excitation amplitude $f$, the resonance frequency increases with $A$. In the nonlinear area, SMA can obviously reduce vibration amplitude, but it is not obvious for the $1 / 3 \mathrm{rd}$ subharmonic resonance. The nonlinear solution of both the $1 / 3 \mathrm{rd}$ subharmonic resonance and 3rd superharmonic resonance are stable.
\end{abstract}

Keywords: SMA laminated beam, subharmonic resonance, superharmonic resonance, transition set

\section{Introduction}

The shape memory alloy (SMA) as an intelligent material with special shape memory and pseudoelasticity, which has been widely applied to mechanics, aerospace, building, civil construction, medicine, chemical, energy, and daily production and life. Zeng and Wan (2011) and Yu (2018) investigated that SMA is often made into composite structures for structural control. SMA laminated structures, such as SMA laminated beams or plates, are fabricated by embedding SMA particles, wires or strips into other matrix materials or covering surfaces of the beam or plate matrices with SMA membranes.

SMAs as surface and linear-elastic materials make up laminated structures, which laminated beams are a common form of. A typical application is a SMA beam structure installed in the

\footnotetext{
${ }^{1}$ These authors contributed equally to this work and should be considered co-first authors.
} 
engine jet of Boeing 777 and 787. Collet et al. (2001) considered a symmetry assumption for SMA under tensile, compressive and temperature loads, and examined dynamic behavior of the material by applying moving external loads to SMA beams. Machado et al. (2003) and Savi et al. (2002) investigated dynamic characteristics and chaotic behavior of coupled SMA oscillators through numerical approaches. In a series of efforts around SMA laminated beams, Ren and Shao (2001), Shao and Ren (2003, 2004), Ren et al. (2010) looked at damping characteristics of laminated beams composed of a mix of SMA and ordinary fibers, and analyzed how the laying angle of fibers and the percentage of SMA in composite beams would affect the equivalent damping ratio of the beams. They also checked the vibration frequency response of structures revealing that, under stress-induced martensite, the hyperelastic nonlinearity of SMA gives rise to complicated dynamics in the structures. They also analyzed the effects of external excitation amplitude and ambient temperature on nonlinear dynamic behavior of the beams. Odeny et al. (2017) studied nonlinearity of aeroelastic behavior of a reinforced SMA hybrid composite (SMAHC) cylindrical plate on a microscopic mechanics model of a carbon fiber-SMAHC laminated plate using the layered Rayleigh-Ritz procedure. Akhavan-Rad and Kheirikhah (2019) applied high-order sandwich panel theory to vibration analysis of an SMA wire-embedded laminated plate and provided an analytical solution to the nonlinear control equation for simply-supported sandwich panels under uniform and sinusoidal loading.

Since the start of constitutive modeling of SMA in the 1980s, a large number of constitutive models, including piecewise-linear model, Brinson's polynomial constitutive model and Falk's model, have been developed to describe the shape memory and pseudoelasticity of SMA. Fu and $\mathrm{Lu}$ (2012) focused on the dynamic response of SMA laminated beams under a piecewise linear resilience model and examined how external excitation amplitude and temperature affected the system response behavior. Lu et al. (2009) proposed a relatively common constitutive SMA fiber constitutive relation and built a bending theory model of SMA fiber-reinforced composite laminated beams without considering the stress relation between the SMA layer and the matrix layer. Zhang (2012) developed a singularity theory for multi-piece description of bifurcations on a Tanaka-Liang-Brinson model by describing the delayed constitutive relation of SMA with a biflag piecewise linear function and investigated nonlinear vibration of SMA laminated beams by building a dynamic model on the basis of their piecewise linear constitutive relation. Asadi et al. (2013) addressed free vibration of SMA fiber-embedded composite laminated beams using Brinson's polynomial constitutive model to consider their stress-strain relation and discussed how temperature variations affected the resilience and fundamental frequency of the beams. Samadpour et al. (2016) built a control equation for laminated beams with SMA fibers that considered the effects of thermal and aerodynamic loads using Brinson's polynomial constitutive model and suggested that the thermal flutter of these beams could be greatly enhanced by embedding SMA fibers, and their stability boundaries could be improved by increasing the volume fraction of SMA fibers. Ghaznavi and Shariyat (2017) observed the nonlinear dynamic behavior or an SMA laminated plate. Razavilar et al. (2018) developed a semi-analytical method to examine free and forced vibration of SMA beams, modeled the control dynamic equation for SMA beams coupled with deformation strain and analyzed dynamic characteristics of these beams using the phase trajectory. Nassiri-Monfared et al. (2018) used a modified Brinson's polynomial constitutive model to investigate the thermodynamic behavior of SMA laminated beams. They involved the potential asymmetric behavior of SMA during tension and compression in their model and recommended a semi-analytical formula. They studied hysteresis loops with their proposed asymmetric model and compared their result with the result of finite analysis.

The resonance includes the main resonance, subharmonic and superharmonic resonance. The resonance has advantages and disadvantages in life, so it is significant to study the resonance phenomenon. Despite all studies in this field, however, few authors have turned their eyes to subharmonic or superharmonic resonances of SMA laminated beams. In practice, exposure to 
periodically variable excitations can greatly affect the system even if the periodic frequency is far from the natural frequency of the system. Conventionally, nonlinear vibration analyses of SMA laminated beams involve a complicated process of using a piecewise model with a hysteresis loop to describe the constitutive relation of SMA. In this paper, we establish a transverse vibration equation for SMA laminated beams under transverse loading using the widely accepted continuously smooth polynomial constitutive model and examined how external excitations and structural parameters (those related to the thickness ratio between the SMA layer and the beam matrix) affect the dynamic characteristics of beams under the $1 / 3 \mathrm{rd}$ subharmonic resonance and the 3rd superharmonic resonance.

\section{Dynamic modeling}

In this study, we used the modified SMA quintic polynomial constitutive model proposed by Falk (Paiva and Savi, 2006), which is structurally simple and convenient for describing the dynamic characteristics of SMA. The stress-strain constitutive relation can be expressed as

$$
\sigma=a\left(T-T_{M}\right) \varepsilon-b \varepsilon^{3}+e \varepsilon^{5}
$$

Here, $\sigma$ represents the stress, $\varepsilon$ represents the strain, and $a, b, T_{A}, T_{M}, e$ are constant parameters. According to Falk (Paiva and Savi, 2006), $T_{A}=313 \mathrm{~K}, T_{M}=287 \mathrm{~K}$ (where $\mathrm{K}$ is the absolute temperature, $T_{A}$ is the austenite transformation temperature, $T_{M}$ is the martensitic transformation temperature), and $a=1 \cdot 10^{3} \mathrm{MPa} / \mathrm{K}, b=40 \cdot 10^{6} \mathrm{MPa}, e=\left[4\left(T_{A}-T_{M}\right) a\right] / b^{2}$. By introducing $a, b, T_{A}, T_{M}$ into the expression of $e$, we have $e=13 /\left(2 \cdot 10^{11}\right) \mathrm{MPa}$.

The structure of a simply-supported laminated beam is shown in Fig. 1, according to Zhang (2012). The matrix in the intermediate layer is constructed of a linear-elastic material-carbon steel with length $L$, thickness $H$, cross-sectional width $d$, and cross-sectional area $d H$. It is sandwiching the beam matrix by two thin SMA layers with equal thickness $h$.

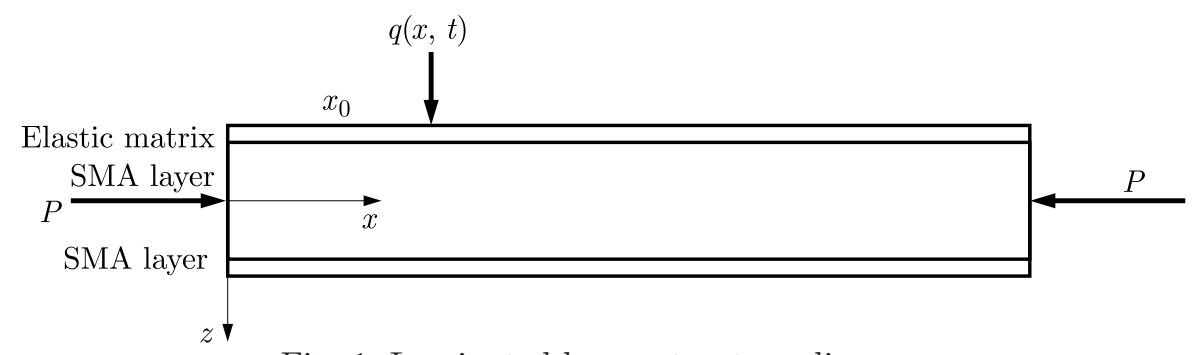

Fig. 1. Laminated beam structure diagram

Assume that the beam is exposed to a concentrated load $f_{0} \sin \lambda t$ at $x_{0}$. Then the periodic concentrated load can be expressed with $\delta$ function as follows

$$
q(x, t)=\delta\left(x-x_{0}\right) f_{0} \sin \lambda t
$$

A dynamic equation for the laminated beams was built with respect to the beam microbody. The loading on the microbody is shown in Fig. 2.

Upper layer (SMA)

$$
h \frac{\partial \sigma_{s 1}}{\partial x}+\tau_{s 1}=0 \quad q(x, t)=p_{s 1}
$$

Intermediate layer (beam matrix)

$$
\begin{array}{ll}
\frac{\partial M}{\partial x}+V-\frac{1}{2} d H\left(\tau_{s 1}+\tau_{s 2}\right)=0 & \tau_{s 1}=\tau_{s 2} \\
m \frac{\partial^{2} w}{\partial t^{2}}=\frac{\partial V}{\partial x}+p_{s 1}-p_{s 2}-c \frac{\partial w}{\partial t} &
\end{array}
$$

where $m$ is mass of the beam matrix per unit length and $c$ is damping per unit length. 


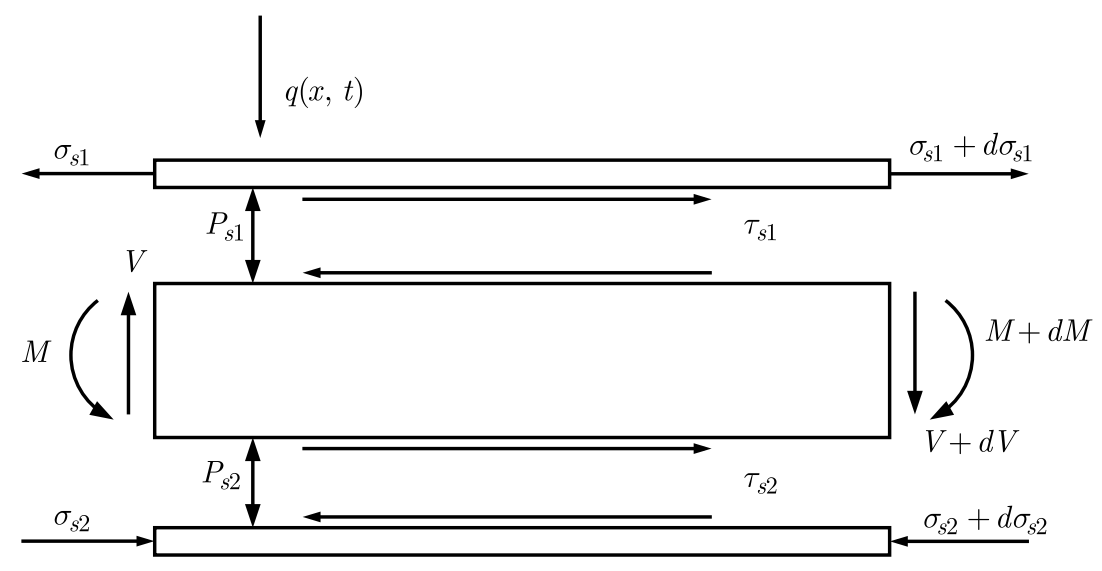

Fig. 2. Force diagram of microbody

Lower layer (SMA)

$$
h \frac{\partial \sigma_{s 2}}{\partial x}-\tau_{s 2}=0 \quad p_{s 2}=0
$$

From the Euler beam theory, we can derive the differential equation for the beam matrix as

$$
M=E \frac{d H^{3}}{12} \frac{\partial^{2} w}{\partial x^{2}}
$$

where $E$ is the elastic modulus of the beam and $d H$ is the sectional area of the beam.

The coordination condition for the displacement between the SMA layer and the beam matrix is

$$
u+\frac{h}{2} \frac{\partial w}{\partial x}=u_{1}-\frac{H}{2} \frac{\partial w}{\partial x} \quad u_{2}+\frac{H}{2} \frac{\partial w}{\partial x}=u-\frac{h}{2} \frac{\partial w}{\partial x}
$$

Only transverse vibration of the beam, rather than the axial force, was examined. Here, $u$ is the axial displacement at individual points on the neutral layer of the beam matrix, consequently, $u=0 ; u_{1}, u_{2}$ are the axial displacements on the symmetric plane of the upper and lower SMA layers, consequently, $u_{1}=-u_{2}$.

On this basis, we get

$$
u_{1}=\frac{H+h}{2} \frac{\partial w}{\partial x}
$$

From Eqs. $(2.3)_{1},(2.4)_{2}$ and $(2.5)_{1}$, we have

$$
\tau_{s}=\tau_{s 1}=\tau_{s 2}=-h \frac{\partial \sigma_{s 1}}{\partial x}=h \frac{\partial \sigma_{s 2}}{\partial x}
$$

And the relation between displacement and strain is

$$
\varepsilon=\frac{\partial u}{\partial x}
$$

Finally, we get the nondimensional normalized dynamic equation for the SMA laminated beam as 


$$
\begin{aligned}
\frac{\partial^{2} \bar{w}}{\partial \bar{t}^{2}} & +\frac{c \tau}{m} \frac{\partial \bar{w}}{\partial \bar{t}}+\frac{E d H_{2}^{3} \tau^{2}}{12 m L} \frac{\partial^{4} \bar{w}}{\partial \bar{x}^{4}}+d H_{1} H_{2}^{2} \frac{\tau^{2}}{m L}\left[E E_{1} \frac{H_{2}\left(1+H_{1}\right)}{2} \frac{\partial \bar{w}^{4}}{\partial \bar{x}^{4}}\right. \\
& -\frac{3}{8} E E_{2} H_{2}^{3}\left(1+H_{1}\right)^{3}\left(\frac{\partial^{2} \bar{w}}{\partial \bar{x}^{2}}\right)^{2} \frac{\partial^{4} \bar{w}}{\partial \bar{x}^{4}}-\frac{3}{4} E E_{2} H_{2}^{3}\left(1+H_{1}\right)^{3} \frac{\partial^{2} \bar{w}}{\partial \bar{x}^{2}}\left(\frac{\partial^{3} \bar{w}}{\partial \bar{x}^{3}}\right)^{2} \\
& \left.+\frac{5}{32} E E_{3} H_{2}^{5}\left(1+H_{1}\right)^{5}\left(\frac{\partial^{2} \bar{w}}{\partial \bar{x}^{2}}\right)^{4} \frac{\partial^{4} \bar{w}}{\partial \bar{x}^{4}}+\frac{5}{8} E E_{3} H_{2}^{5}\left(1+H_{1}\right)^{5}\left(\frac{\partial^{2} \bar{w}}{\partial \bar{x}^{2}}\right)^{3}\left(\frac{\partial^{3} \bar{w}}{\partial \bar{x}^{3}}\right)^{2}\right] \\
& =\frac{\tau^{2}}{m L} \delta\left(\bar{x}-x_{0}\right) f_{0} \sin \lambda \bar{t}
\end{aligned}
$$

where

$$
\begin{aligned}
& \bar{x}=\frac{x}{L} \quad \bar{w}=\frac{w}{L} \quad H_{1}=\frac{h}{H} \quad H_{2}=\frac{H}{L} \quad \frac{a\left(T-T_{M}\right)}{E}=E_{1} \\
& \frac{b}{E}=E_{2} \quad \frac{e}{E}=E_{3} \quad \bar{t}=\frac{t}{\tau} \quad \tau=\frac{2}{\pi^{2} H_{2}} \sqrt{\frac{3 m L}{H_{2} E b}}
\end{aligned}
$$

\section{Amplitued-frequency response equation}

The displacement response of the beam can be expressed as

$$
\bar{w}=Y(\bar{t}) \sin \pi \bar{x}
$$

To make computation easier, the axial strain is used to indicate deflection of the beam, $Y(\bar{t})$. From Eq. (2.8), we can derive the strain distribution as

$$
\varepsilon_{s}=\frac{\partial \bar{u}_{s}}{\partial \bar{x}}=\frac{H_{2}\left(1+H_{1}\right)}{2} \frac{\partial^{2} \bar{w}}{\partial \bar{x}^{2}}=x(\bar{t}) \sin \pi \bar{x}
$$

where $x(\bar{t})$ is the strain at the midpoint of the beam. By introducing Eq. (3.1) into Eq. (3.2), we have

$$
Y(\bar{t})=-\frac{2 x(\bar{t})}{n^{2} \pi^{2} H_{2}\left(1+H_{1}\right)}
$$

again, by introducing $Y(\bar{t})$ and (3.1) into (2.11) and order $c \tau / m=c_{0}, E d \tau^{2} /(m L)=G$. According to the nature of $\delta$ function and through the Galerkin process, we derive the dynamic equation for the transverse vibration of the laminated beam as

$$
\ddot{x}+x=-c_{0} \dot{x}-6 E_{1} A x+\frac{9}{2} E_{2} A x^{3}-\frac{15}{4} E_{3} A x^{5}+f \sin \lambda t
$$

where

$$
\begin{array}{lrrr}
f=\frac{f_{0} \tau^{2}}{m L} \sin \pi x_{0} & E=206 \mathrm{GPa} & c_{0}=0.1 & H_{1}\left(1+H_{1}\right)=A \\
E_{1}=\frac{T-287}{206} & E_{2}=\frac{20000}{103} & E_{3}=\frac{10^{8}}{1339}
\end{array}
$$

Conclusions regarding the primary resonance of these systems are provided in Zhang and $\mathrm{Wu}$ (2017). In practical application, however, more complicated nonlinearities will occur in a nonlinear system when exposed to multi-frequency excitations. In the following Sections, we are going to explore further into the $1 / 3$ rd subharmonic resonance and the 3rd superharmonic resonance of SMA laminated beams. 


\subsection{The $1 / 3$ rd subharmonic resonance of the system}

Assume the relation between the external excitation frequency and periodic natural frequency to be $\omega=\lambda / 3+\varepsilon \sigma$. Then the primary approximate solution can be expressed as

$$
x=y \cos \left(\frac{1}{3} \lambda t+\theta\right)=y \cos \psi \quad \dot{x}=-y \omega \sin \left(\frac{1}{3} \lambda t+\theta\right)=-y \sin \psi
$$

Using the averaging method, we can derive the averaged equation of the system as

$$
\begin{aligned}
& \frac{d y}{d t}=-\frac{1}{2} c_{0} y-\frac{1}{6} f \cos 3 \theta \\
& \frac{d \theta}{d t}=1-\frac{1}{3} \lambda+3 A E_{1}-\frac{27}{16} E_{2} A n^{2} y^{2}+\frac{75}{64} E_{3} A n^{2} y^{4}+\frac{1}{6 y} f \sin 3 \theta
\end{aligned}
$$

Order $d y / d t=d \theta / d t=0$. Then the amplitude-frequency response equation of the system under the $1 / 3 \mathrm{rd}$ subharmonic resonance is

$$
\frac{9 c_{0}^{2} y^{2}}{f^{2}}+\frac{36 y^{2}}{f^{2}}\left(\frac{1}{3} \lambda-1-3 A E_{1}+\frac{27}{16} E_{2} A y^{2}-\frac{75}{64} E_{3} A y^{4}\right)^{2}=1
$$

\subsection{The 3rd superharmonic resonance of the system}

For the 3rd superharmonic resonance, we assume the relation between the external excitation frequency and periodic natural frequency to be $\omega=3 \lambda+\varepsilon \sigma$. Then the primary approximate solution can be expressed as

$$
x=y \cos (3 \lambda t+\theta)=y \cos \psi \quad \dot{x}=-y \omega \sin (3 \lambda t+\theta)=-y \sin \psi
$$

Using the averaging method, we can derive the averaged equation of the system as

$$
\begin{aligned}
& \frac{d y}{d t}=-\frac{1}{2} c_{0} y-\frac{3}{2} f \cos \frac{1}{3} \theta \\
& \frac{d \theta}{d t}=1-3 \lambda+3 A E_{1}-\frac{27}{16} E_{2} A n^{2} y^{2}+\frac{75}{64} E_{3} A n^{2} y^{4}+\frac{3}{2 y} f \sin \frac{1}{3} \theta
\end{aligned}
$$

Order $d y / d t=d \theta / d t=0$. Then the amplitude-frequency response equation for the system under the $3 \mathrm{rd}$ superharmonic resonance is

$$
\frac{c_{0}^{2} y^{2}}{9 f^{2}}+\frac{4 y^{2}}{9 f^{2}}\left(3 \lambda-1-3 A E_{1}+\frac{27}{16} E_{2} A y^{2}-\frac{75}{64} E_{3} A y^{4}\right)^{2}=1
$$

External excitation amplitudes $f$ and $A\left(H_{1}\left(1+H_{1}\right)=A\right)$, response amplitude $y$, excitation frequency $\lambda$ are included in both Eq. (3.6) and Eq. (3.9). Each individual parameter has different effects on the system response. These effects could be further studied by carrying out singularity analysis, finding transition set curves, defining parametric spaces, or qualitatively classifying amplitude-frequency response curves.

\section{Transition sets and amplitude-frequency plottings}

The parametric effects of the $1 / 3 \mathrm{rd}$ subharmonic resonance and the $3 \mathrm{rd}$ superharmonic resonance can be further examined by finding the transition sets through singularity theory, taking the external excitation amplitudes $f$ and $A\left(H_{1}\left(1+H_{1}\right)=A\right)$ as the unfolding parameters, the response amplitude $y$ as the state variable, and excitation frequency $\lambda$ as the bifurcation parameter. 
The SMA layer is relatively thin so that we discuss the dynamic characteristics of laminated beams within a smaller range of $A$. According to (3.3), vibration of the laminated beams is weakly nonlinear. So, we can use the weakly nonlinear correlation theory and the Euler beam theory to solve the problem.

Assume the bifurcation equation to be

$$
G(y, \lambda, f, A)=\frac{c_{0}^{2} y^{2}}{9 f^{2}}+\frac{4 y^{2}}{9 f^{2}}\left(3 \lambda-1-3 A E_{1}+\frac{27}{16} E_{2} A y^{2}-\frac{75}{64} E_{3} A y^{4}\right)^{2}-1
$$

Bifurcation point set: $B=\left\{f, A \in R^{k} \mid G=G_{y}=G_{\lambda}=0\right\}$.

Hysteresis point set: $H=\left\{f, A \in R^{k} \mid G=G_{y}=G_{y y}=0\right\}$.

Double limit point set: $\left.D=\left\{f, A \in R^{k} \mid G\left(y_{i}, \lambda, f, A\right)=G\left(y_{i}, \lambda, f, A\right), \quad i=1,2\right), \quad y_{1} \neq y_{2}\right\}$.

As calculated, the bifurcation point set $B$ and the double limit point set $D$ are empty sets; and the hysteresis point set $H$ is non-empty as shown in Fig. 3.

\subsection{Transition sets of the primary resonance, subharmonic resonance, and superharmonic resonance}

As shown in Fig. 3, if $T=300 \mathrm{~K}$, the transit sets of the primary resonance, 1/3rd subharmonic resonance, and 3rd superharmonic resonance are each a hysteresis point set curve. The hysteresis point set curve of the $1 / 3 \mathrm{rd}$ subharmonic resonance shows a "right displacement" and that of the 3rd superharmonic resonance shows a "left displacement", relative to the hysteresis point set curve of the primary resonance.

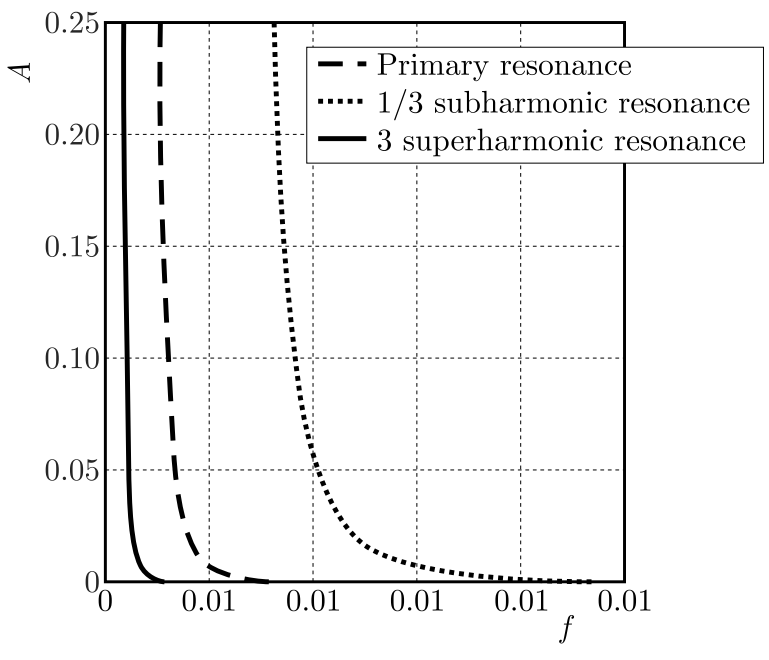

Fig. 3. Transition sets

Figures 4 and 5 compare the amplitude-frequency response curves between the primary resonance, 1/3rd subharmonic resonance, and 3rd superharmonic resonance for the same SMA thickness $A$ and excitation amplitude $f$. From left to right, the dotted line is the amplitude-frequency response curve of the 3rd superharmonic resonance, the solid line is the amplitude-frequency response of the subharmonic resonance, and the double hyphen is that of the $1 / 3 \mathrm{rd}$ subharmonic resonance. Under the same $A$ and $f$, the 3 rd superharmonic resonance displayed the highest resonance amplitude and the greatest vibration followed by the primary resonance. The resonance amplitude was remarkably smaller when the excitation frequency approximated three times the natural frequency of the system. 


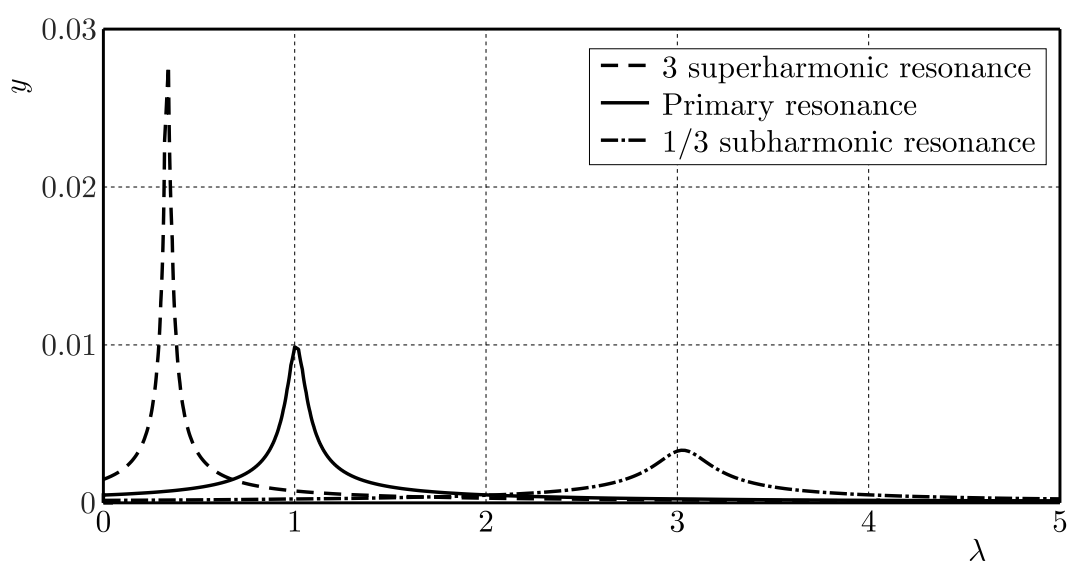

Fig. 4. Amplitude-frequency response diagram; $f=0.001, A=0.05$

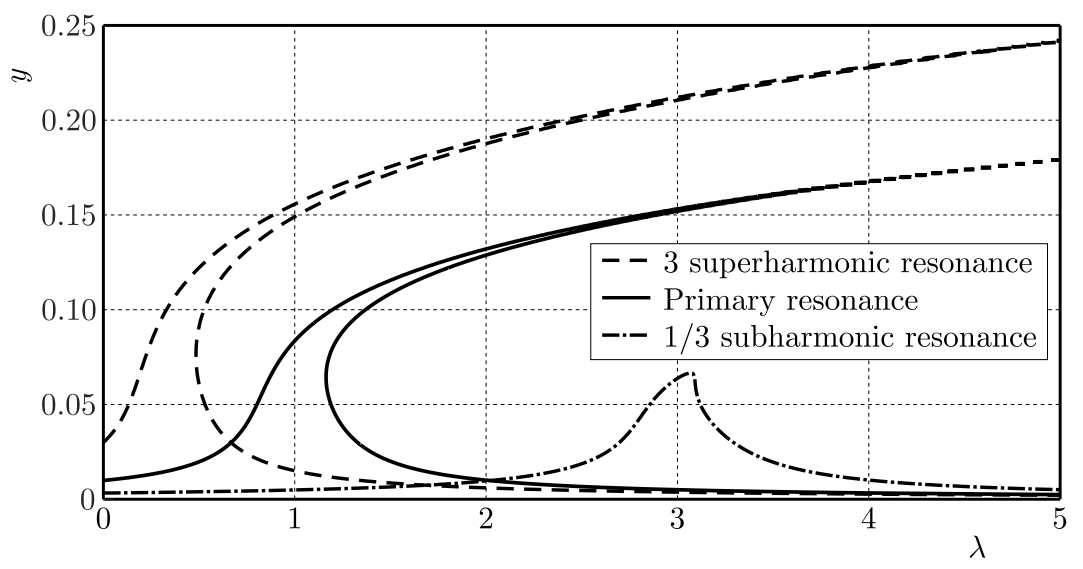

Fig. 5. Amplitude-frequency response diagram; $f=0.02, A=0.05$

\subsection{The transition set and amplitude-frequency response plotting under the $1 / 3 \mathrm{rd}$ subharmonic resonance}

As shown in Fig. 3, the transition set of the 1/3rd subharmonic resonance, namely, the hysteresis point set $H$, separates the parametric region into two subregions within each of which the amplitude-frequency response curves of the system are qualitatively the same. When the parameters $f$ and $A$ fall within the left subregion, these curves appear to be quasi-linear and the resonance frequency occurs at around $\lambda=3$. When the parameters fall within the right subregion, the amplitude-frequency response curves become noticeably nonlinear, with a multisolution zone, which is a sign of a hysteresis, in the right solution branch, showing hard characteristics.

Figure 6 compares the amplitude-frequency response curves under the same $A$ but different $f$. Under a constant SMA thickness, when the external excitation amplitude $f$ increases, the parameters migrate from the left subregion through the hysteresis point set into the right subregion. The response amplitude of the system increases and shows a noticeable hysteresis.

Figures 7, 8 and 9 compare the amplitude-frequency response curves under the same $A$ but different $f$. As shown in Fig. 7, in the quasi-linear area, under the same external excitation amplitude $f$, the level of $A$ does not make much difference to the response of the system. As the SMA thickness increases, the resonance frequency shows a slight "left displacement" signifying a decrease in the resonance frequency. In the nonlinear area, under the same external excitation 


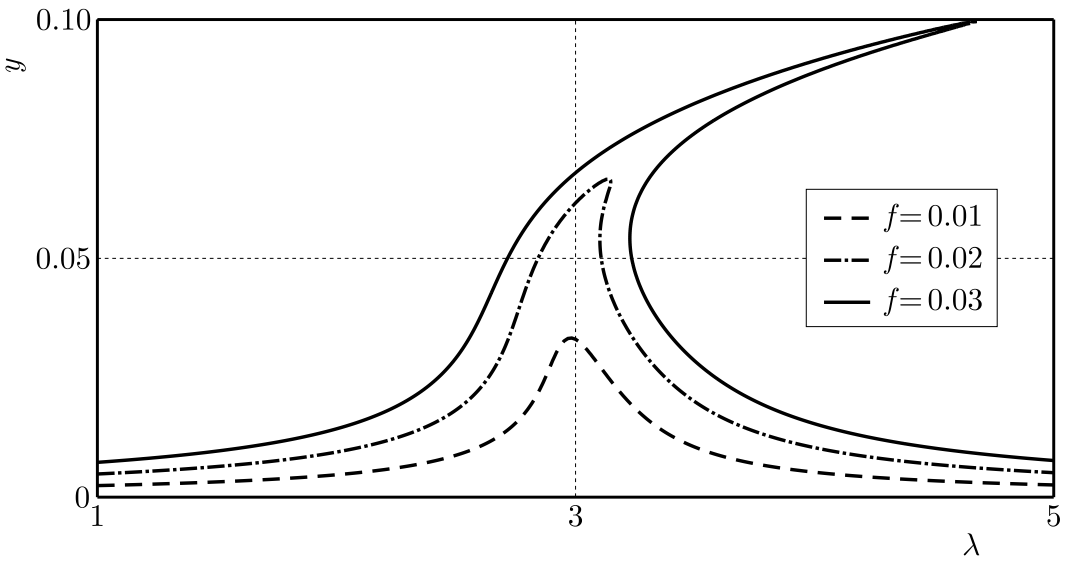

Fig. 6. Amplitude-frequency response diagram; $A=0.1$

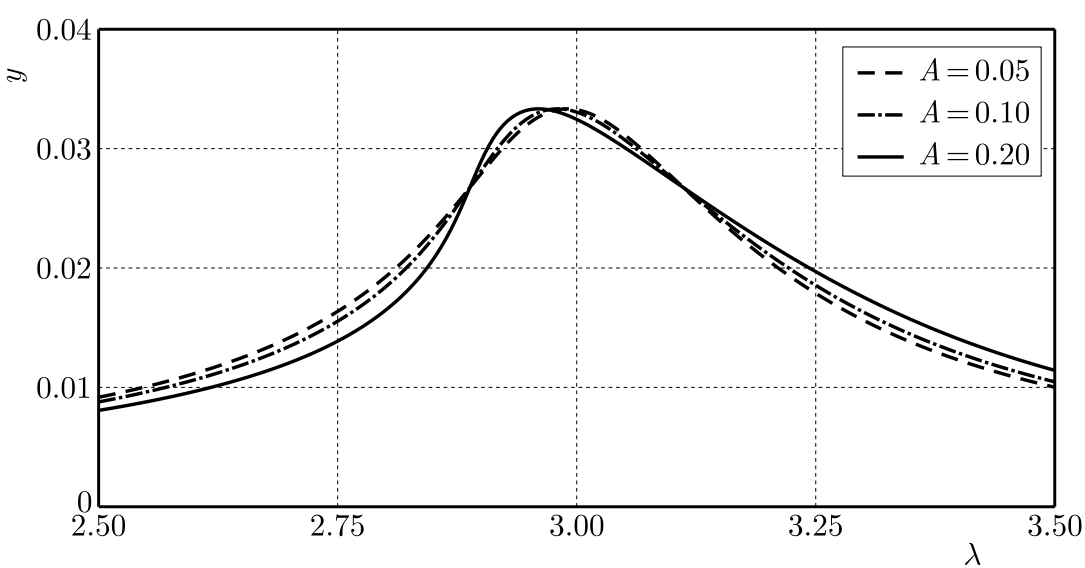

Fig. 7. Amplitude-frequency response diagram; $f=0.01$

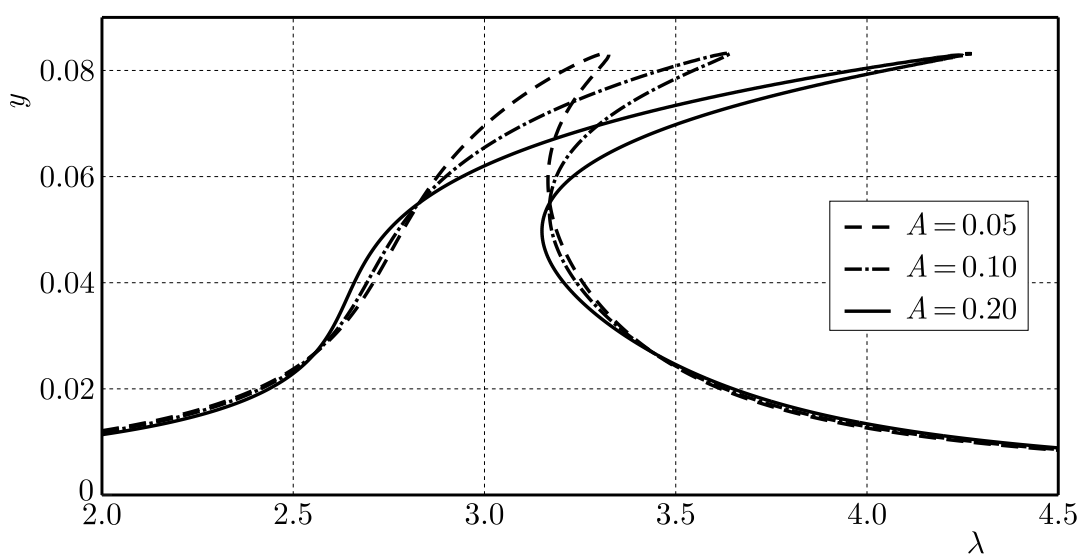

Fig. 8. Amplitude-frequency response diagram; $f=0.025$

amplitude $f$, as $A$ increases, the SMA thickness increases, too, the resonance frequency translates in the direction of the $\lambda$ increase, and the resonance frequency band also broadens, as shown in Fig. 8. As $A$ increases, the response amplitude decreases, especially where $f$ is high, as shown in Fig. 9. 


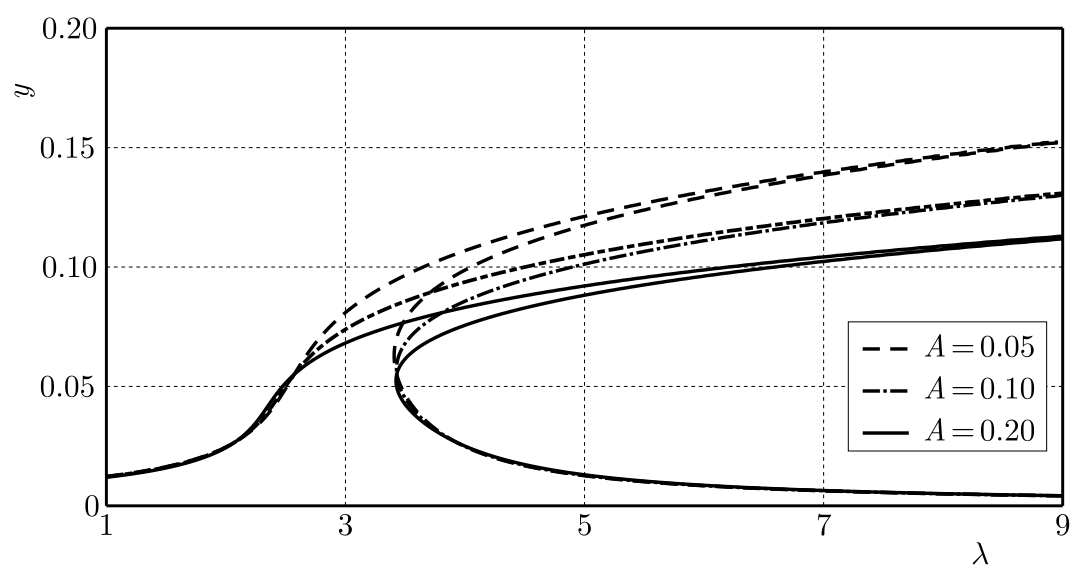

Fig. 9. Amplitude-frequency response diagram; $f=0.05$

\subsection{The transition and amplitude-frequency response plotting under the 3rd superharmonic resonance}

The transition set of the 3rd superharmonic resonance is represented by the black dotted line in Fig. 3. When the parameters $f$ and $A$ fall within the left subregion, the amplitude-frequency response of the system is quasi-linear and the response frequency occurs at around $\lambda=1 / 3$. When these parameters fall within the right subregion, the amplitude-frequency response curve shows a right displacement with a multisolution area, which is a sign of hysteresis, in the right solution branch, showing hard characteristics, as shown in Fig. 10. Furthermore, when the SMA thickness $A$ remains the same, as the external excitation intensity $f$ increases, the response amplitude increases and the hysteresis becomes greater.

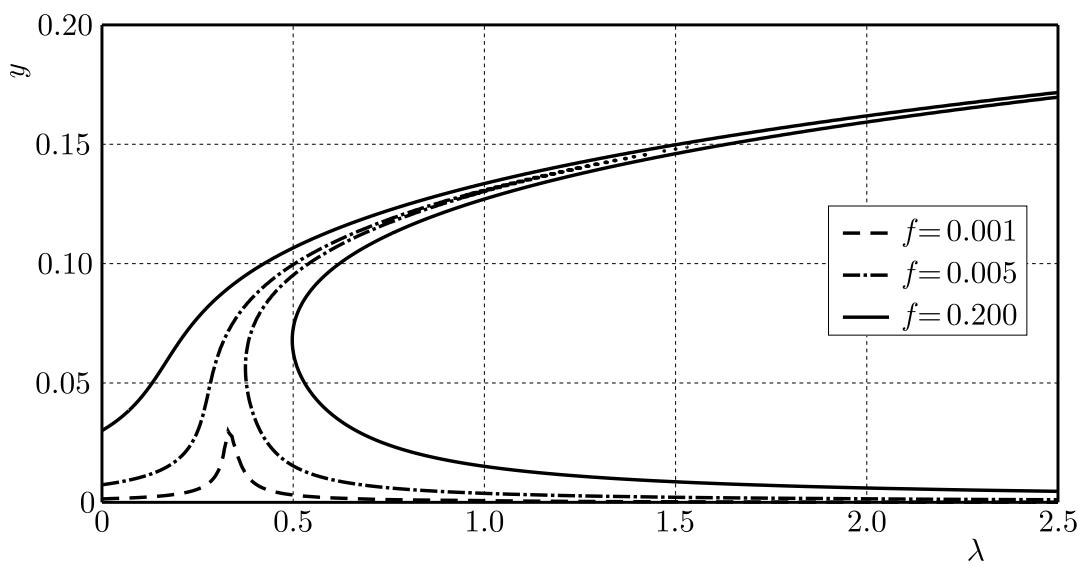

Fig. 10. Amplitude-frequency response diagram; $A=0.1$

By comparing the SMA thicknesses under the same external excitation amplitude, we can see that when $f$ remains the same in the left subregion of the transit set, the level of $A$ does not make much difference to the response of the system, as shown in Fig. 11. This concurs with the conclusion in Fig. 7 for the 1/3rd subharmonic resonance. By comparing Figs. 12 and 13, we can see that, in the nonlinear area, under the same external excitation amplitude $f$, as $A$ increases, the resonance amplitude band broadens and the response amplitude decreases, verifying the vibration reduction by the SMA layer. 


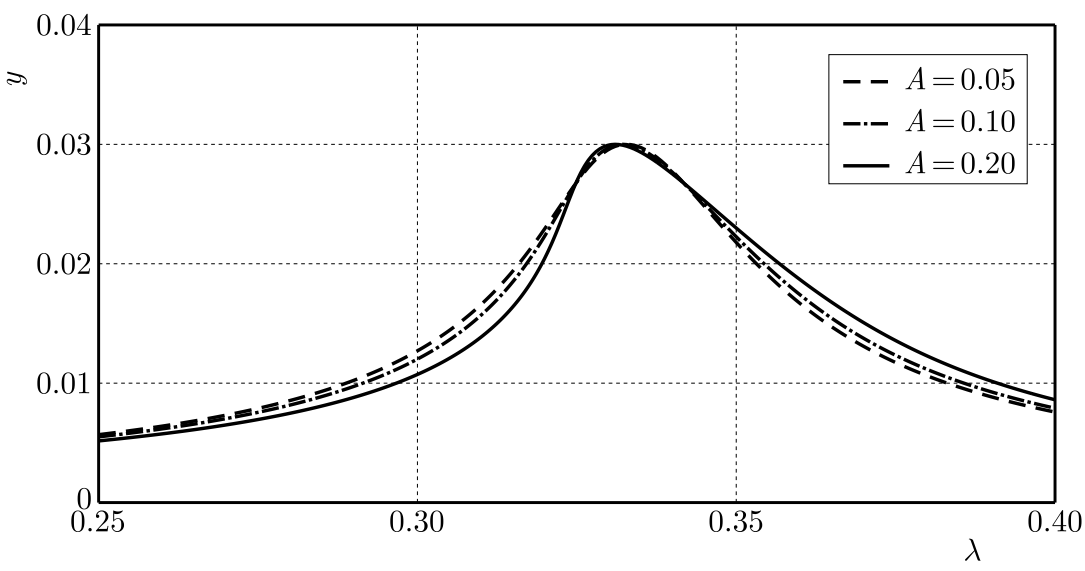

Fig. 11. Amplitude-frequency response diagram; $f=0.001$

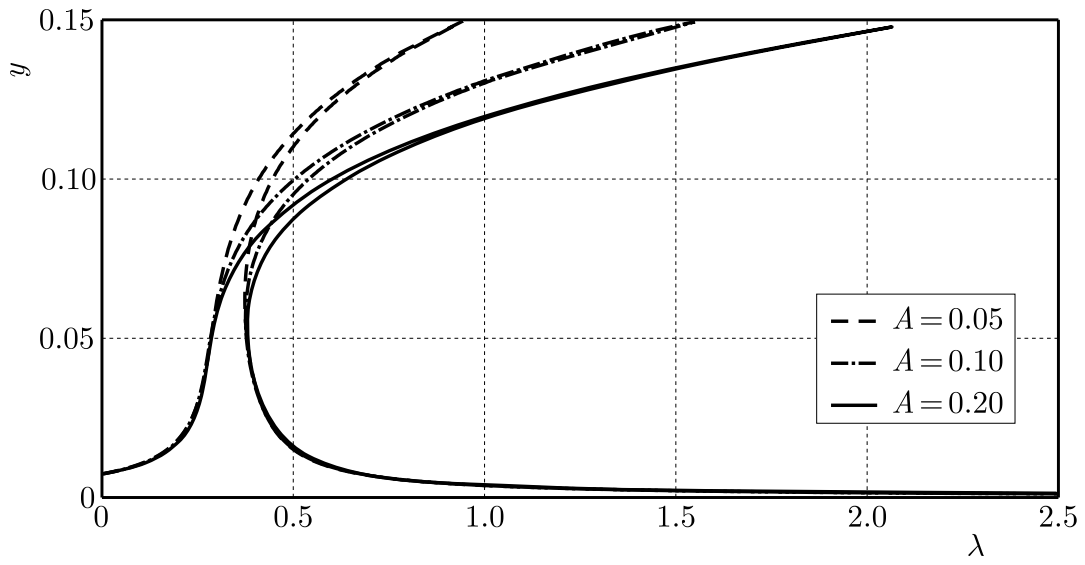

Fig. 12. Amplitude-frequency response diagram; $f=0.005$

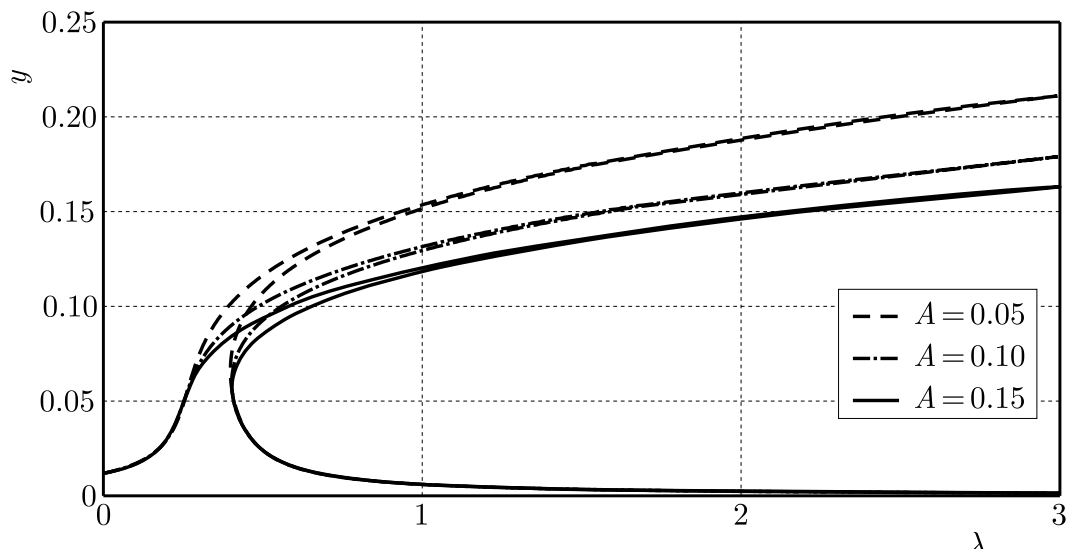

Fig. 13. Amplitude-frequency response diagram; $f=0.008$

\section{Comparison of beams without SMA and the stability analysis}

To analyze the influence of SMA on the beams, Figs. 14 and 15 separately compare the amplitude-frequency response curves between three resonances above $(A=0.05)$ and resonances of beams without SMA $(A=0)$ under different $f$. The colored lines show the amplitudefrequency response diagrams of beams without SMA. As shown in Fig. 14, in the quasi-linear area, under the same external excitation amplitude, SMA does not make much difference to the three resonances of the system. As shown in Fig. 15, in the nonlinear area, with the addi- 
tion of SMA layer, the amplitude of the beam decreases, SMA can obviously reduce vibration amplitude, but the vibration reduction is not obvious for the $1 / 3 \mathrm{rd}$ subharmonic resonance.

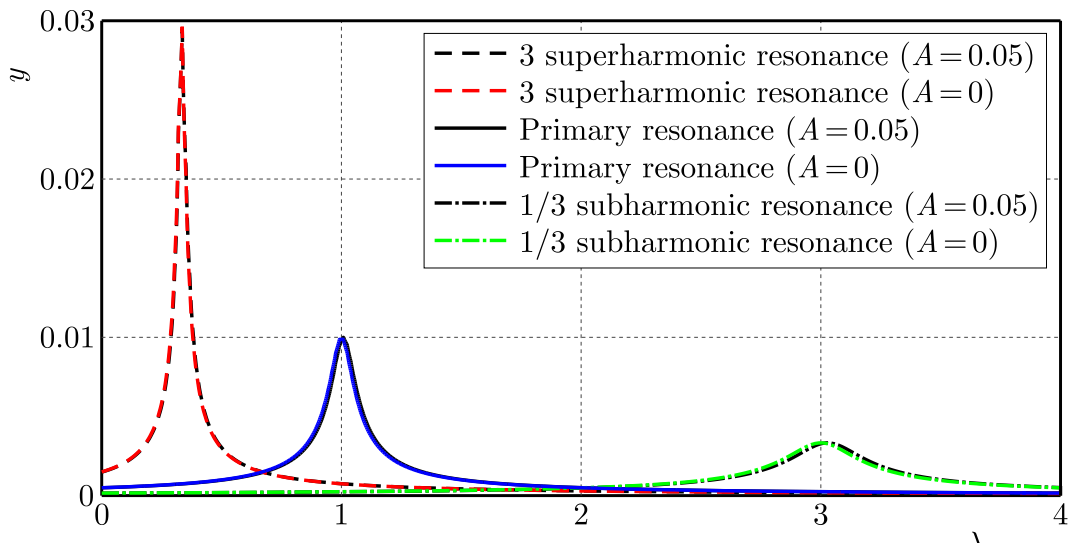

Fig. 14. Amplitude-frequency response diagram; $f=0.001$

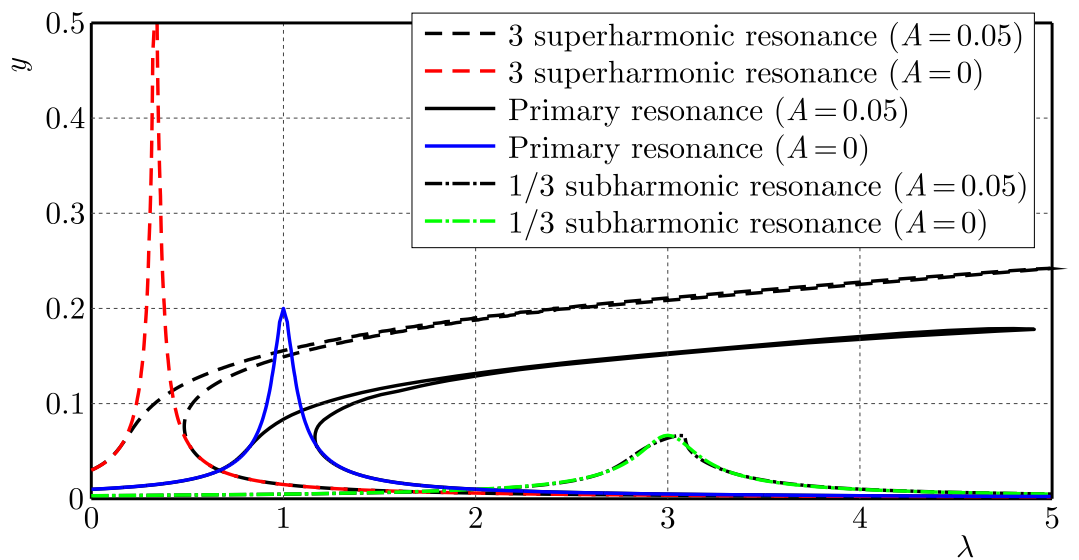

Fig. 15. Amplitude-frequency response diagram; $f=0.02$

To analyze stability of the system, Fig. 16 shows each phase diagram of three resonances, each phase diagram is closed, so the nonlinear solution is stable.

\section{Conclusions}

This paper examines the 1/3rd subharmonic and 3rd superharmonic resonance of SMA laminated beams. The following conclusions are drawn:

- The transition sets of both the 1/3rd subharmonic and 3rd superharmonic resonance are simply a hysteresis point set. The amplitude-frequency response of the system to nonlinear vibration occurs in two forms: quasi-linear and hard characteristics.

- Regarding the two types of nonlinear resonance, increasing the external excitation amplitude $f$ will lead to an increase in the response amplitude of the system in all cases. In the quasi-linear area, the level of $A$ or SMA thickness does not make much difference to the response of the system. In the hard characteristic response, under the same external excitation amplitude $f$, as $A$ increases, the resonance frequency band broadens. In the hard characteristic response, higher excitation amplitudes will mean greater vibration reductions by the SMA layer.

- Under the same $A$ and $f$, the 3rd superharmonic resonance displays the highest vibration and the greatest resonance amplitude followed by the primary resonance. The $1 / 3 \mathrm{rd}$ subharmonic resonance shows a significantly smaller resonance amplitude. 
(a)

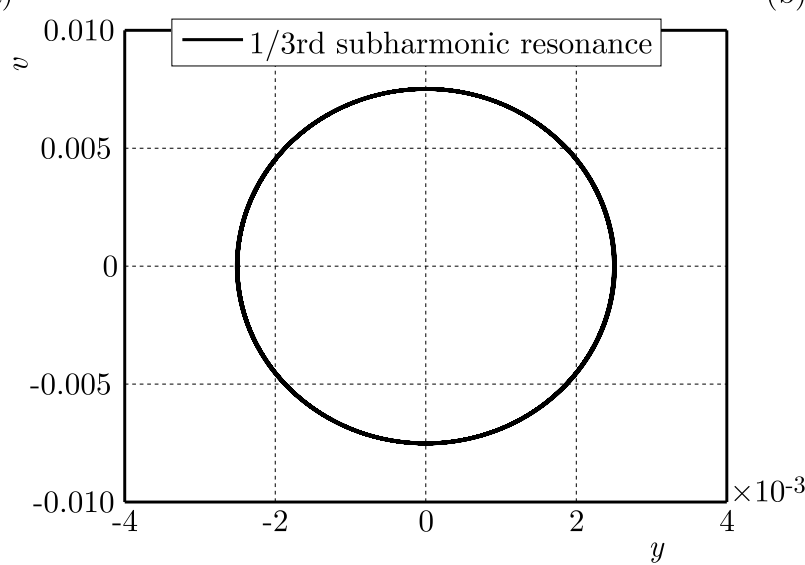

(b)

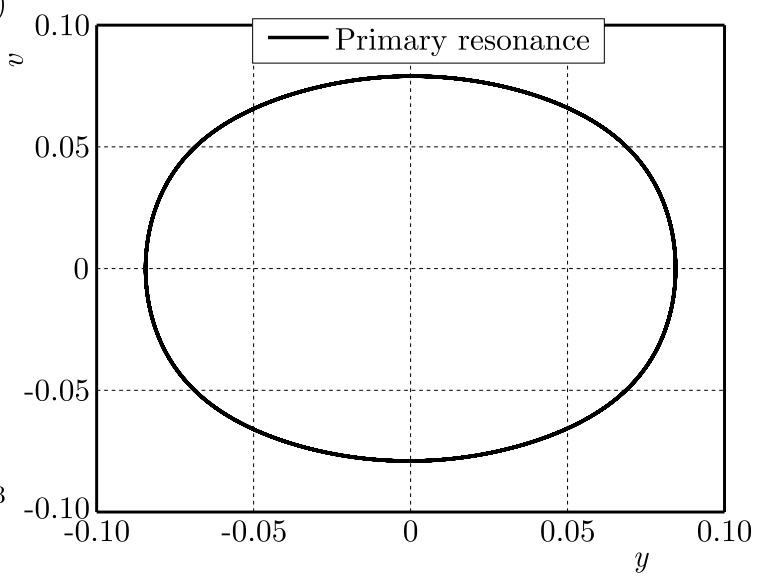

(c)

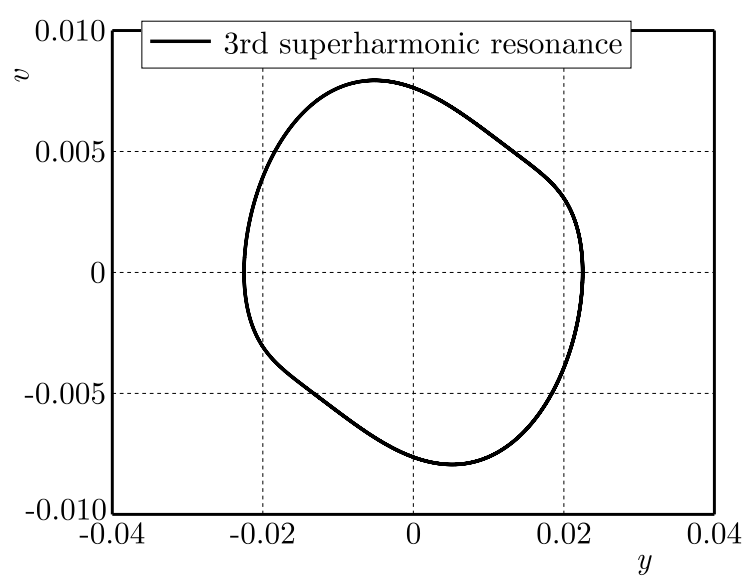

Fig. 16. Phase diagram of velocity and displacement; $f=0.02, A=0.05$

- By comparing three resonances and resonances of beams without SMA, under the same external excitation amplitude, in the quasi-linear area, SMA does not make much difference to the three resonances of the system. In the nonlinear area, SMA can obviously reduce vibration amplitude, but the vibration reduction is not obvious for the $1 / 3 \mathrm{rd}$ subharmonic resonance.

The vibration generated by the resonance can be utilized in some cases, which brings a lot of convenience to life. Similarly, in some cases, the vibration caused by the resonance can cause great harm and should be prevented. Many structures in engineering can be regarded as beams, so it is significant to study the main resonance, the subharmonic and superharmonic resonance of beams. The structure of laminated beams in this paper is also a common composite form of the shape memory alloy. A typical application is the SMA beam structure installed in the engine jet of Boeing 777 and 787, which is composed of SMA and the original material to reduce vibration and noise. In this paper, the matrix in the intermediate layer is constructed of a linear-elastic material-carbon steel, so this method also applies to other linear-elastic materials. And the rest of the calculation is dimensionless, so the conclusions are general. However, the conclusions are true only when SMA layer is relatively thin. The conclusions of this paper provide a theoretical basis and reference for structural design and application as well as research of SMA. The conclusions also provide references for the study of nonlinear characteristics and vibration reduction of SMA, including the application to engineering and aerospace. And our further work is to analyze the internal resonance of the laminated beam. 


\section{Acknowledgements}

The authors acknowledge the National Natural Science Foundation of China (Grant No. 11902287, No. 32072786), the Natural Science Foundation of Shandong Province (Grant No. ZR2018MEE001, No. ZR2018MC017), the Natural Science Foundation of Tianjin (Grant No. 19JCYBJC19100), the Open Project Program of Tianjin Key Laboratory of Microgravity and Hypogravity Environment Simulation Technology (Grant No. TJWDZL2019KT004).

\section{References}

1. Akhavan-Rad B., Kheirikhah M.M., 2019, Static analysis of sandwich plates embedded with shape memory alloy wires using active strain energy tuning method, Journal of the Brazilian Society of Mechanical Sciences and Engineering, 41,160, 1-17

2. Asadi H., Bodaghi M., Shakeri M., Aghdam M.M., 2013, On the free vibration of thermally pre/post-buckled shear deformable SMA hybrid composite beams, Aerospace Science and Technology, 31, 73-86

3. Collet M., Foltête E., Lexcellent C., 2001, Analysis of the behavior of a shape memory alloy beam under dynamical loading, European Journal of Mechanics - A/Solids, 20, 4, 615-630

4. FU S., LU Q., 2012, Nonlinear dynamics and vibration reduction of a dry friction oscillator with SMA restraints, Nonlinear Dynamics, 69, 3, 1365-1381

5. Ghaznavi A., Shariyat M., 2017, Non-linear layerwise dynamic response analysis of sandwich plates with soft auxetic cores and embedded SMA wires experiencing cyclic loadings, Composite Structures, 171, 185-197

6. Lu P., CUi F.S., TAN M.J., 2009, A theoretical model for the bending of a laminated beam with SMA fiber embedded layer, Composite Structures, 90, 4, 458-464

7. Machado L.G., Savi M.A. Pacheco P.M.C.L.,, 2003, Nonlinear dynamics and chaos in coupled shape memory oscillators, International Journal of Solids and Structures, 40, 19, 5139-5156

8. Nassiri-Monfared A., Baghani M., Zakerzadeh M.R., Fahimi P., 2018, Developing a semi-analytical model for thermomechanical response of SMA laminated beams, considering SMA asymmetric behavior, Meccanica, 53, 4-5, 957-971

9. Odeny D.M. Jr, Donadon M.V., Castro S.G.P., 2017, Aeroelastic behavior of stiffened composite laminated panel with embedded SMA wire using the hierarchical Rayleigh-Ritz method, Composite Structures, 181, 26-45

10. Paiva A., Savi M.A., 2006, An overview of constitutive models for shape memory alloys, Mathematical Problems in Engineering, 2006, 4, 1-30

11. Razavilar R., Fathi A., Dardel M., Hadi J.A., 2018, Dynamic analysis of a shape memory alloy beam with pseudoelastic behavior, Journal of Intelligent Material Systems and Structures, 29, 9, 1835-1849

12. Ren Y., Liu Y., Yang S., Wang X., 2010, Active deformation models of SMA fiber hybrid thin-walled laminated beams, Chinese Journal of Solid Mechanics, 31, 3, 228-236

13. Ren Y., Shao B., 2001, Material damping of SMA fiber hybrid laminated beam, Mechanics in Engineering, 23, 16-20

14. Samadpour M., Asadi H., Wang Q., 2016, Nonlinear aero-thermal flutter postponement of supersonic laminated composite beams with shape memory alloys, European Journal of Mechanics - A/Solids, 57, 18-28

15. Savi M.A., Pacheco P.M.C.L., Braga A.M.B., 2002, Chaos in a shape memory two-bar truss, International Journal of Non-Linear Mechanics, 37, 8, 1387-1395

16. Shao B., Ren Y., 2003, Analysis of free vibrations of shape memory alloy hybrid composite beams, Engineering Mechanics, 20, 4, 183-187 
17. Shao B., Ren Y., 2004, The semi-active control of shape memory alloy composite beam, Mechanics in Engineering, 26, 16-19

18. Yu H., 2018, New application of shape memory alloy on variable thickness wing, Internal Combustion Engine and Parts, 17, 234-235

19. Zeng S., WAn X., 2011, Shape memory alloys and their application in aviation industry (1), Heat Treatment Technology and Equipment, 32, 3, 1-5

20. Zhang Z., 2012, Dynamic bifurcation and control of the structures with Shape Memory Alloy (SMA), Ph.D. Thesis, Tianjin University, Tianjin, China

21. Zhang X., Wu Z., 2017, Bifurcation analysis of shape memory alloy laminated beam, Chinese Journal of Applied Mechanics, 34, 3, 397-403

Manuscript received May 21, 2019; accepted for print May 3, 2020 